\title{
Influences of Network Delay and Moving Velocity on Virtual Cooperative Work with Haptic Sense
}

\author{
May Zin $\mathrm{Oo}^{1+}$, Yutaka Ishibashi ${ }^{2}$, Khin Than $\mathrm{Mya}^{3}$ \\ ${ }^{1,3}$ University of Computer Studies, Yangon, Myanmar \\ ${ }^{2}$ Nagoya Institute of Technology, Nagoya, Japan
}

\begin{abstract}
In this paper, we investigate influences of the network delay and moving velocity on virtual cooperative work with haptic sense by experiment. In the work, each of two users operates a haptic interface device, and the two users collaboratively raise a stick in a 3D virtual space. The stick has a weighted ball, and the ball moves to the lower end along the stick if one end of the stick is lower than the other end. They try to keep the ball at the center of the stick while raising the stick. Experimental results demonstrate that it is possible to keep the ball around at the center when the network delay is small. We also show that there exists the optimal moving velocity of the ball depending on the network delay.
\end{abstract}

Keywords: virtual cooperative work, haptic sense, network delay, moving velocity, experiment.

\section{Introduction}

A number of researchers have been studying networked virtual environments with haptic sense [1]-[4]. We can largely improve the efficiency of collaborative work by using haptic sense. In such work, it is necessary for multiple users to do collaborative work while watching the same displayed images in a 3D virtual space simultaneously. However, when the information about the space is transmitted over a Quality of Service (QoS) [5] non-guaranteed network like the Internet, the receiving times of the information at different terminals may be different from each other owing to network delays, delay jitters, and so on. That is, some of the terminals may have already received information while the others may have not received the information yet. Then, the efficiency of collaborative work may deteriorate seriously because users at the terminals may watch different displayed images at the same time.

To solve the above problem, as QoS control, we need to perform simultaneous output-timing control [6], which absorbs delay differences among different terminals. Various types of the control such as the local lag control [7], the group synchronization control [8], and the adaptive $\Delta$-causality control [9] can be employed.

In [2], Arimoto et al. deal with a networked music ensemble system with haptic sense. A pair of users play drums. In the system, the network delay and packet loss degrade collaboration performance between the users. They examine the influence of network delay, the effect of media synchronization control, and the role of haptic sense in the system. As a result, they illustrate that using haptic sense in addition to the visual and auditory senses can enhance the collaboration performance and improve the sense of togetherness among individuals taking part in the work. However, they investigate only the influence of network delay in the work; they do not examine influences of other factors such as the velocity of hitting drums.

In [4], the authors handle a networked balance system with haptic sense in which each of two users operates a haptic interface device, and the two users collaboratively raise a stick by lifting two ends of the stick up in a 3D virtual space. The stick has a weighted ball, and the ball moves to the lower end along the stick if one end of the stick is lower than the other end. They try to keep the ball at the center of the stick

\footnotetext{
+ Corresponding author. Tel.: +81-76-288-8110
}

E-mail address: mayzinoo2018@ucsy.edu.mm 
while raising the stick. They carry out the local lag control as simultaneous output-timing control, and they make a comparison between results with the local lag control and those without the control to clarify the effect of the control on the human perception of weight. However, the influences of the other factors such as the moving velocity of the ball on human perception of weight have not been investigated. We need to examine influences of the moving velocity as well as the network delay on the collaborative work to carry out QoS control more efficiently.

In this paper, we deal with the networked balance system with haptic sense [4], and we investigate the influences of the network delay and moving velocity on the work efficiency. We also examine the influence of the initial position of the ball.

The remainder of this paper is organized as follows. Section 2 outlines the networked balance system with haptic sense, and Section 3 describes the experiment method. Then, Section 4 presents experimental results and discusses them. Finally, Section 5 concludes the paper.

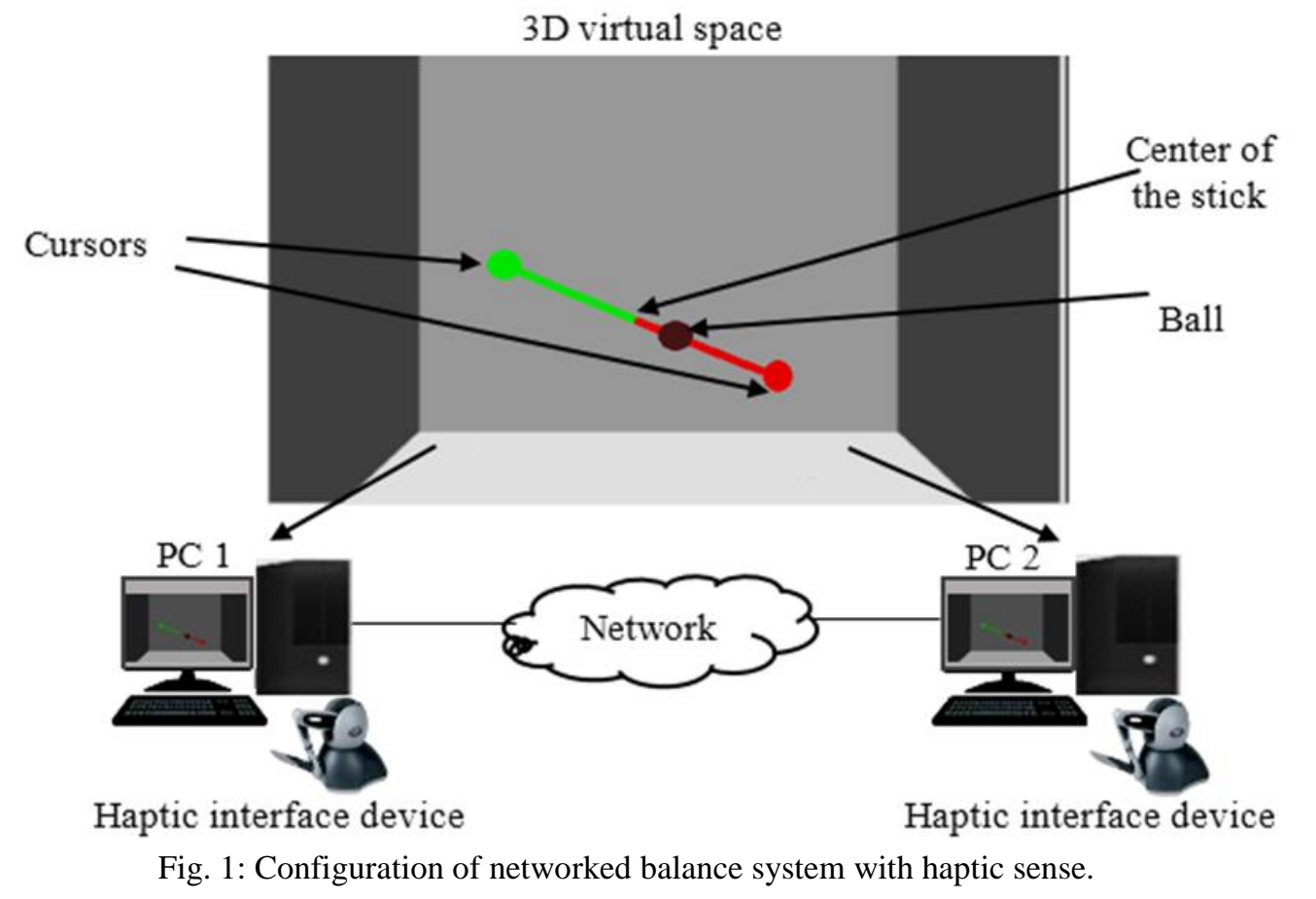

\section{Networked Balance System with Haptic Sense}

We show the configuration of the networked balance system with haptic sense and a displayed image of the 3D virtual space in Fig. 1. The system consists of two terminals (called PCs 1 and 2 here) each of which has a haptic interface device (3D Systems Touch [10]).

By operating the haptic interface device, a user of each PC can move a cursor which denotes the position of the device stylus's tip in the space which is surrounded by a floor, a ceiling, and walls. The two cursors are fixed at two ends of a stick whose weight is 0 gf. The stick can be stretched or shrunk freely between the two ends. A ball whose weight is $270 \mathrm{gf}$ is placed on the stick between the two cursors (i.e., the two ends of the stick). The reaction force applied to a user is proportional to the distance from the user's cursor to the ball (as shown in the 3D virtual space of Fig. 1, the right side of the center is green, and the left side of the center is red). We show the calculation method of reaction force in Fig. 2, where the proportion of the distance from the red cursor to the ball to the distance from the green cursor to the ball is set to $k:(1.0-k)$. Also, $m$ is the mass of the ball, and $\boldsymbol{g}\left(9.8 \mathrm{~m} / \mathrm{s}^{2}\right)$ is the gravitational acceleration. The reaction force applied to the red cursor's user is $(1.0-k) \boldsymbol{F}$, and that applied to the green cursor's user is $k \boldsymbol{F}$. The ball moves towards the lower cursor along the stick if there exist altitude differences between the two cursors. In this paper, we assume that there is no viscous resistance. The users try to keep the ball at the center of the stick while lifting up the stick. 


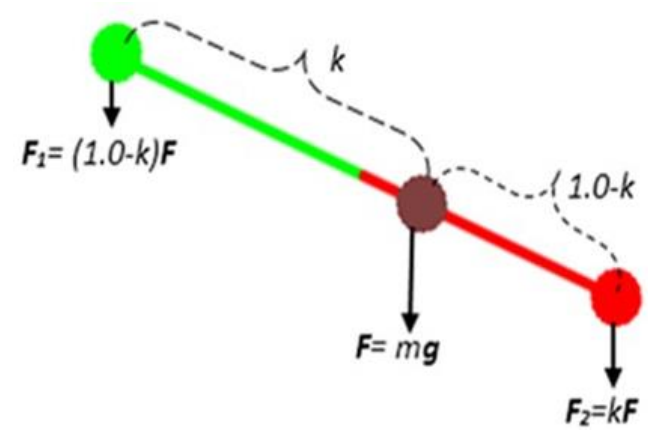

Fig. 2: Calculation method of reaction force.

\section{Experiment Method}

In our experiment system, we employed a network emulator (netem [11]) instead of the network in Fig. 1 to generate a constant delay (called the additional delay in this paper) for each packet transmitted between the two PCs. Note that we here handle constant delay for simplicity because media synchronization control absorbs the fluctuation of the network delay to some extent. In the experiment, we handled two methods (called Methods 1 and 2). In Method 1, the users started to lift up the ball from the initial state in which the weighted ball was placed at the center of the stick, and the two cursors were placed on the floor (see Fig. 3 (a)). The initial position of the ball in Method 2 is different from that in Method 1; the ball was placed at the far left of the stick (see Fig. 3 (b)). In both methods, the users were asked to lift the ball up to a height of 16.7 (we assume that the diameter of cursor is 1 in this paper) collaboratively while trying to keep the ball at the center of the stick and to move at a constant velocity (see Fig. 4). The movement distance of 16.7 corresponds to $10 \mathrm{~cm}$ in the real space when each user raises the stylus of the haptic interface device vertically.

If the ball was not located at the center, each user tried to adjust the place of the ball by moving up his/her cursor or stop moving. Then, we investigated the influence of the additional delay and moving velocity, and we also examine whether the optimal velocity exists for each additional delay.

We carried out the experiment in which the additional delay from PC 1 to PC 2 was set to the same value as that from PC 2 to PC 1, and the additional delay was changed from $0 \mathrm{~ms}$ to $150 \mathrm{~ms}$ at intervals of $50 \mathrm{~ms}$. Also, we changed the moving velocity from 0.1 to 0.9 at intervals of 0.2 and from 1.0 to 13.0 at intervals of 2.0.

The positions of the ball and the operation time were measured in the experiment. We define the position of the ball as the distance between the ball and the center of the stick. Positions on the right side of the center are denoted by plus values, and those on the left side are done by minus ones. The operation time is defined as a time interval from the moment one user starts to raise the cursor until the instant one user's cursor reaches the maximum height of 16.7 .

In the experiment, in both methods, the combinations of the additional delay and moving velocity were selected in random order for each work. The experiment was conducted by two users (females) whose ages were 35 . We carried out the experiment 10 times.

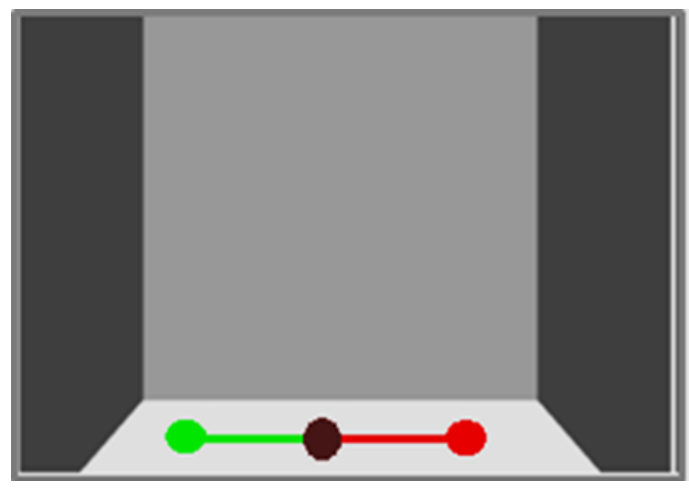

(a) Ball at center (Method 1)

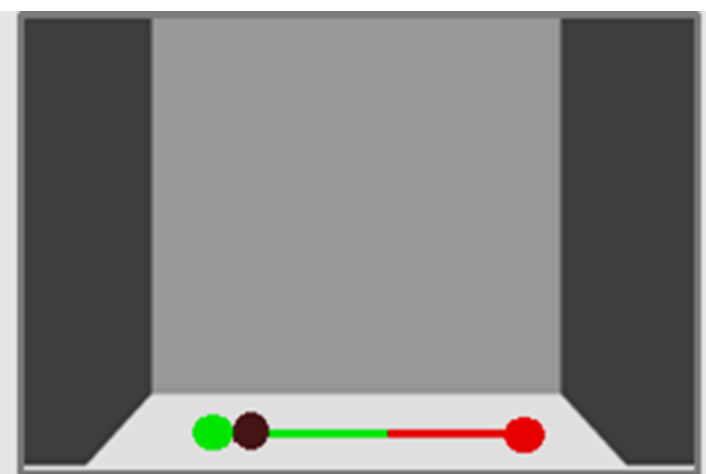

(b) Ball at far left (Method 2)

Fig. 3: Displayed images of initial state in experiment. 


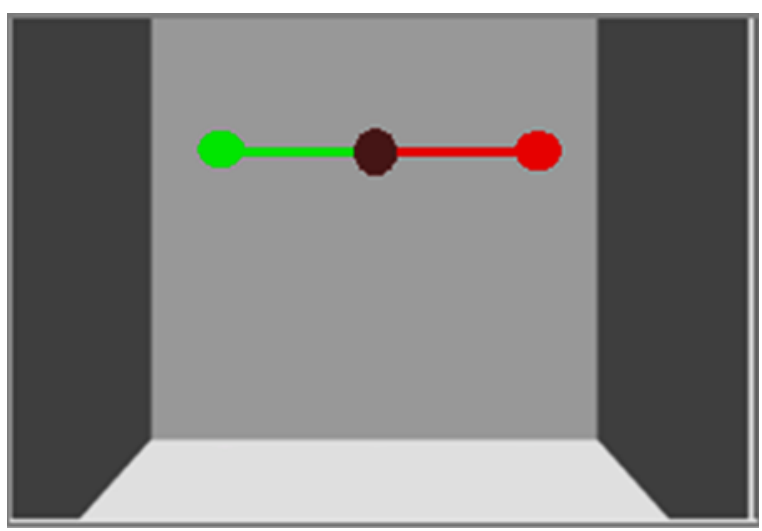

Fig. 4: Displayed image of end state in experiment.

\section{Experimental Results}

We show the average of average absolute position (i.e., the mean of 10-times average absolute position) versus the moving velocity at PC 1 and PC 2 in Figs. 5 (a) and (b), respectively, for Method 1. Figure 6 shows the average operation time versus the additional delay for Method 1. Figures 7 (a) and (b) plot the average of average absolute position versus the moving velocity at PCs 1 and 2, respectively, for Method 2 . Figure 8 shows the average operation time versus the additional delay for Method 2.

In Fig. 5, we see that in Method 1, as the additional delay and moving velocity increase, the average of average absolute position becomes larger. This means that it is difficult to keep the ball at the center of the stick when the moving velocity is faster than around 10 . We also notice in the figure that the average of average absolute position is smaller than about 2 for the velocity less than around 4 ; in this case, the ball was almost kept at the center. We further observe that the results of PC 1 are somewhat different from those of PC 2. We can adjust the positions at PCs 1 and 2 by performing simultaneous output-timing control; this is our further study.

From Fig. 6, we find that the average operation time increases as the moving velocity and additional delay become larger. Therefore, the users tried to adjust the ball many times when the moving velocity and additional delay were large.

From Fig. 7, we notice that there exists the optimal moving velocity for each additional delay in Method 2. It should be noted that the optimal moving velocity has the smallest average of average absolute position. Thus, the users can more easily adjust the ball at the center of the stick at the optimal velocity. This is because when the moving velocity is too small, it takes a long time to move the ball to the center, and when the moving velocity is too large, it is difficult to keep the ball at the center of the stick. It should be noted that smaller values of the moving velocity are better in Method 1. Furthermore, we confirm in Fig. 7 that the results of PC 1 are largely different from those of PC 2 when the moving velocity is larger than about 3.0.

Figure 8 reveals that the average operation time becomes shorter as the moving velocity increases. This is because we can easily keep the ball at the center by lifting the cursors up timely when the initial position of the ball is far left; that is, the user at PC 1 lifts the cursor at first, and then the user at PC 2 lifts the cursor while watching the ball so that the ball can stops at the center. Therefore, the influence of the initial position of the ball on the operation time is large.

\section{Conclusions}

In this paper, we investigated the influences of the network delay and moving velocity on the work efficiency in the networked balance game where two users collaboratively lift up a weighted ball with haptic sense in a virtual environment. We also examined the influence of the initial position of the ball. As a result, we found that it is possible to keep the ball around at the center when the network delay is small. Also, there exists the optimal moving velocity depending on the network delay.

As our next step, we will examine influences of other factors such as the weight and size of the ball on the work efficiency. It is also important to make a mathematical model of our system and to analyze the results in this paper. 


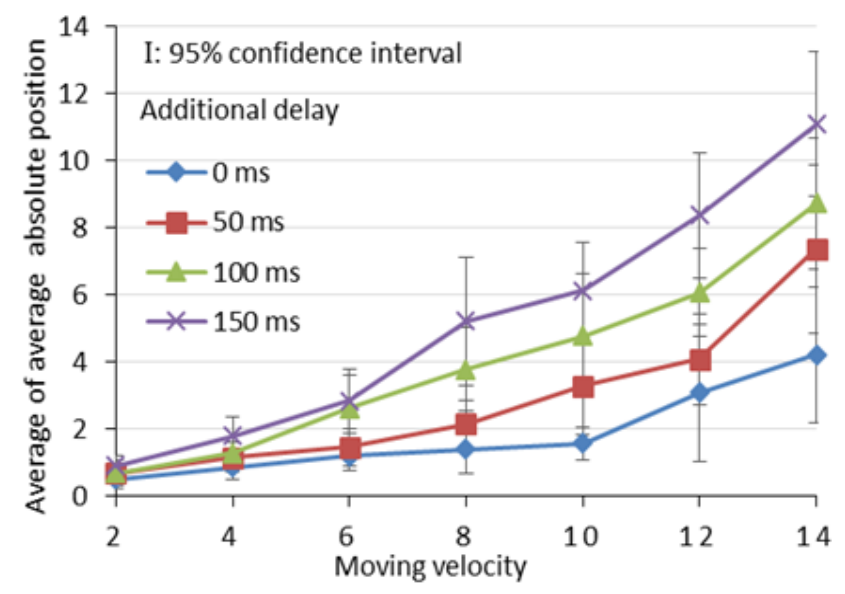

(a) PC 1

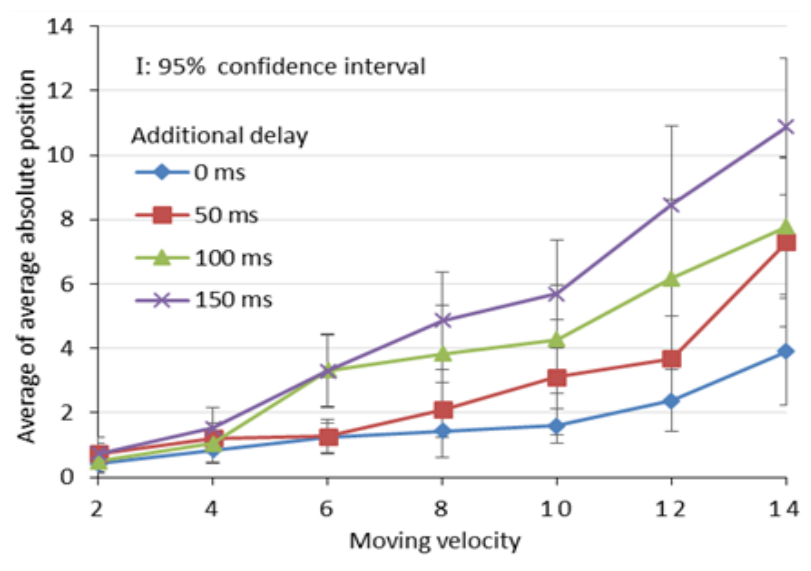

(b) $\mathrm{PC} 2$

Fig. 5: Average absolute position versus moving velocity in Method 1.

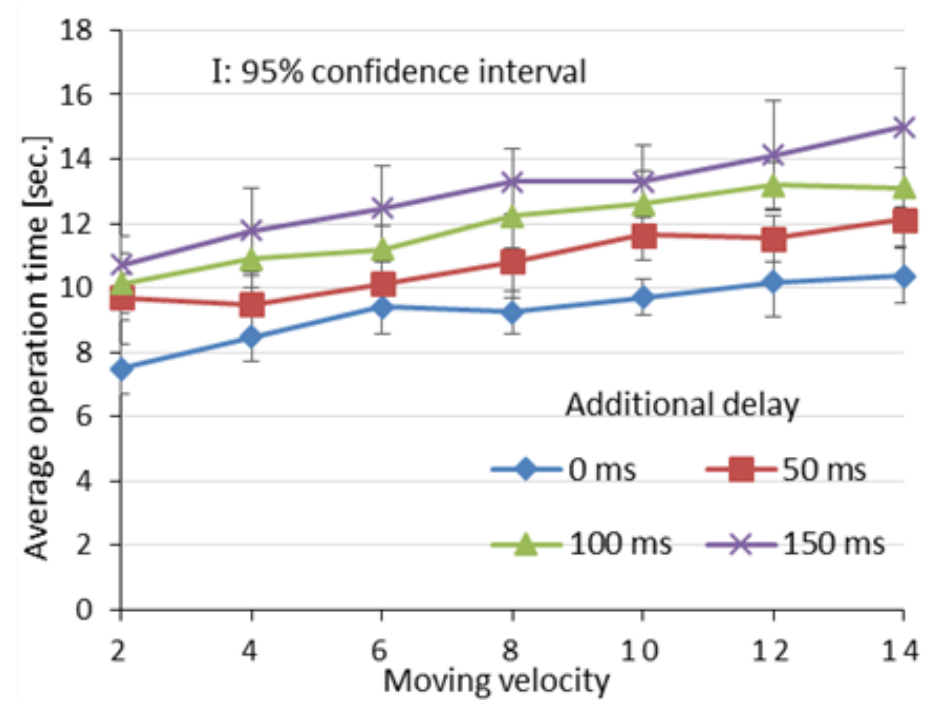

Fig. 6: Average operation time versus moving velocity in Method 1.

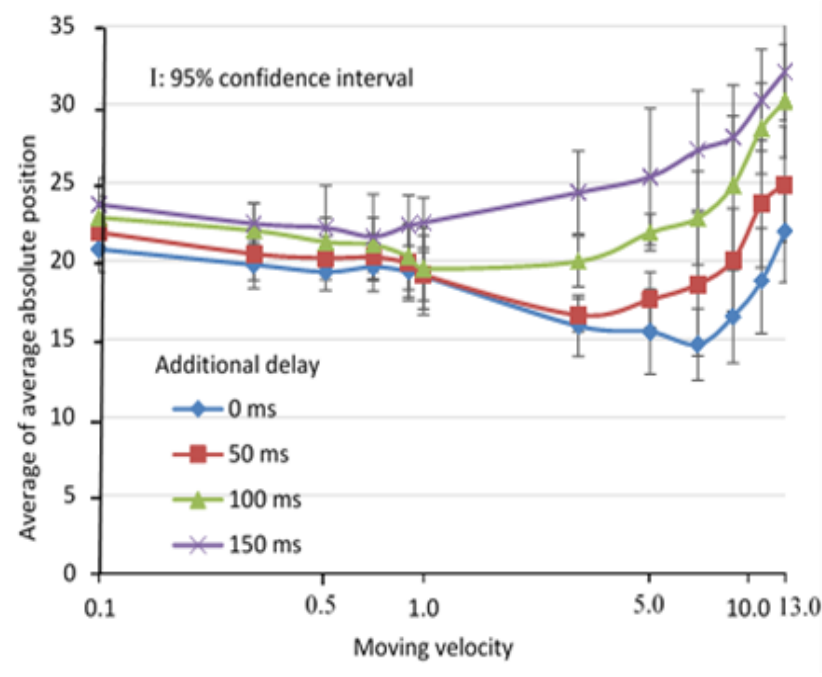

(a) PC 1

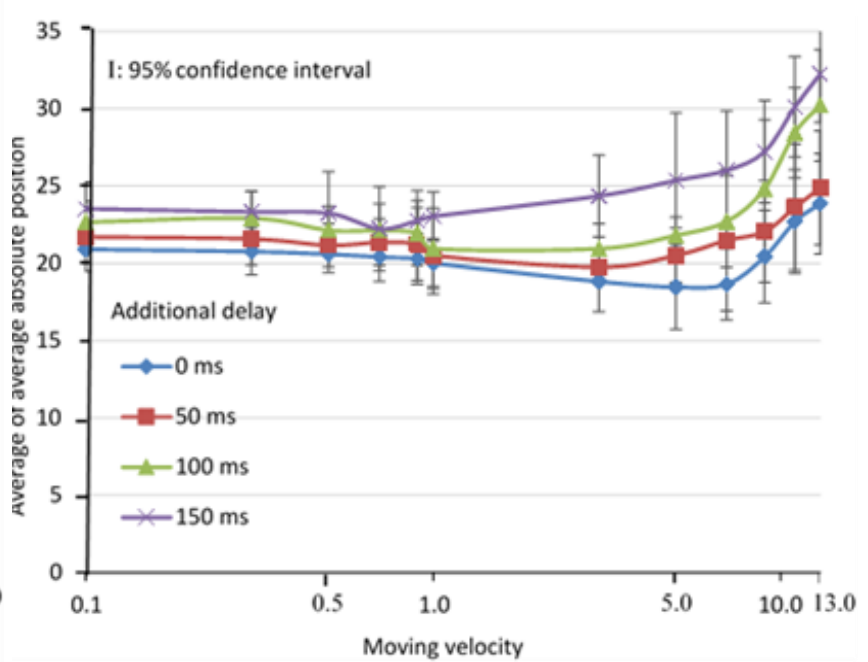

(b) PC 2

Fig. 7: Average of average absolute position versus moving velocity in Method 2. 


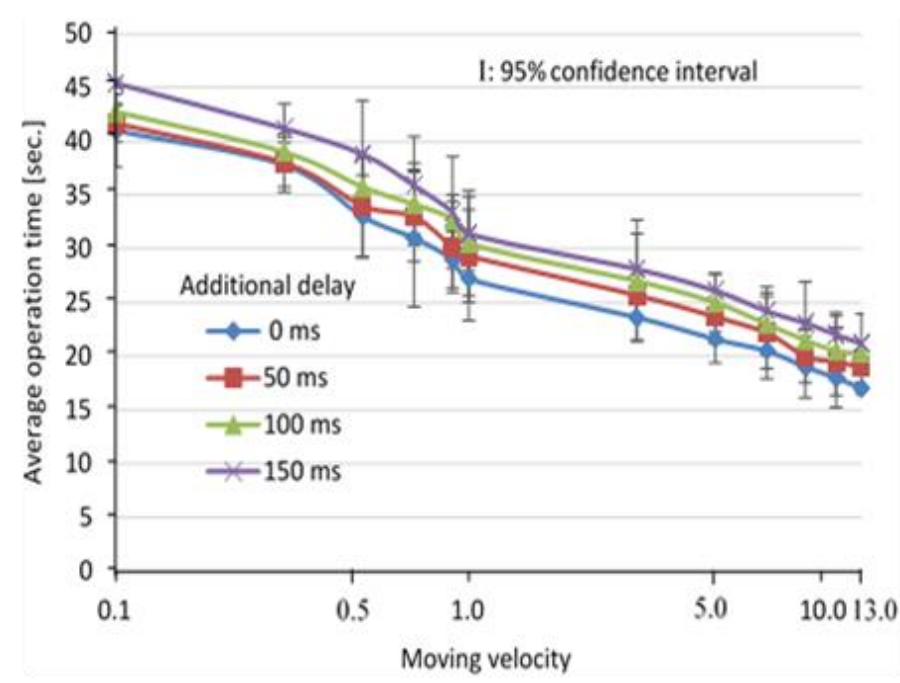

Fig. 8: Average operation time versus moving velocity in Method 2.

\section{References}

[1] E. Steinbach, S. Hirche, M. Emst, F. Brandi, R. Chaudhari, J. Kammerl, and I. Vittorias, "Haptic communications," IEEE Journals \& Magazines, vol. 100, no. 4, pp. 937-945, Apr. 2012.

[2] I. Arimoto, K. Hikichi, K. Sezaki, and Y. Yasuda, "Influence of network delay on ensemble application" in Proc. IEEE International Workshop on Haptic Audio Visual Environments and their Applications (HAVE), pp. 1-2, Oct. 2005.

[3] B. M. Lambeth, J. LaPlant, E. Clapan, and F. G. Hamza-Lup, "The effects of network delay on task performance in a visual-haptic collaborative environment," in Proc. ACM The 47th Annual Southeast Regional Conference, pp. 1-5, Mar. 2009.

[4] P. Huang and Y. Ishibashi, "Human perception of weight in networked virtual environment with haptic sense: Influence of network delay,” Journal of Communications (JCM), vol. 14, no. 6, pp. 478-483, June 2019.

[5] ITU-T Rec. I. 350 "General aspects of quality of service and network performance in digital networks," Mar. 1993.

[6] P. Huang, Y. Ishibashi, and M. Sithu, "Enhancement of simultaneous output-timing control with human perception of synchronization errors among multiple destinations," in Proc. The 2nd IEEE International Conference on Computer and Communications (ICCC), pp. 2099-2103, Oct. 2016.

[7] M. Mauve, J. Vogel, and W. Effelsberg, "Local lag and timewarp: Providing consistency for replicated continuous applications," IEEE Trans. on Multimedia, vol. 6, no. 1, pp. 47-57, Feb. 2004.

[8] Y. Ishibashi, A. Tsuji, and S. Tasaka, "A group synchronization mechanism for stored media in multicast communications," in Proc. IEEE International Conference on Computer Communications (ICCC), pp. 693-701, Apr. 1997.

[9] Y. Ishibashi, S. Tasaka, and Y. Tachibana, "Adaptive causality and media synchronization control for networked multimedia applications," in Conf. Rec. IEEE International Conference on Communications (ICCC), pp. 952-958, June 2001.

[10] [Online] Available: https://www.3dsystems.com/haptics-devices/touch.

[11] [Online] Available: http://www.linux.org/man8/tc-netem. 